\title{
A Simple Score to Predict Fetal Outcomes in Gestational Diabetes Mellitus
}

\author{
Kushal Naha, Sowjanya Naha, Vinay R. Pandit, Shubha Seshadri
}

Background: Strict glycemic control is critical in preventing adverse maternal and fetal outcomes with gestational diabetes mellitus (GDM), but frequently results in recurrent maternal hypoglycemia and is often impracticable. This study was done to determine whether a more lenient strategy might provide satisfactory outcomes and to formulate a glycemic score for prognostication of fetal outcomes.

Methods: A prospective non-interventional study was conducted on consecutive patients admitted with GDM between May 2007 and August 2009. Patients with pre-gestational diabetes were excluded. All patients received treatment at the discretion of treating consultants. Glycemic control was estimated by recording mean values of all glucose profiles performed. Fasting and postprandial blood glucose levels below $95 \mathrm{mg} / \mathrm{dl}$ and $120 \mathrm{mg} / \mathrm{dl}$, respectively, were considered controlled. A glycemic score was calculated based on the number of mean blood glucose values controlled. Fetal outcomes were noted.

Results: Ninety-four patients with GDM were included. The glycemic score was significantly predictive of adverse fetal outcomes $(p<0.001)$. Analysis by receiver operating characteristic (ROC) curve showed good sensitivity and specificity for macrosomia $(78.3 \%$ and $81.8 \%$, respectively) and congenital anomalies (73.9\% and $66.7 \%$, respectively) with a glycemic score of 2 or less [area under curve (AUC) 0.768; odds ratio (OR), 11.17; 95\% Confidence Interval (CI), 2.58-48.35; $p<0.001$; and AUC 0.765; OR, 2.22; 95\% CI, 0.71-6.92; $p=0.055$, respectively]. Binomial logistic regression confirmed the glycemic score to be independently predictive of fetal outcome ( $p=0.015)$.

Conclusion: The glycemic score is a sensitive and specific prognostic marker. Tight control of three of four values of blood glucose within the glucose profile appears sufficient to prevent adverse fetal outcomes.

(Biomed J 2015;38:131-135)

\section{At a Glance Commentary}

Scientific background of the subject

Gestational diabetes mellitus (GDM) is a state of carbohydrate intolerance with onset or first recognition during pregnancy, and complicates up to $14 \%$ of all pregnancies. GDM has been linked with maternal mortality, fetal death and malformation, and long-term sequelae such as increased risk for development of type 2 diabetes in the mother and cardio-metabolic risk factors in the child. The rigorous glycemic control required to prevent these complications entails self-monitoring of blood glucose, compliance with diet, and pharmacologic therapy, and runs the risk of recurrent hypoglycemia - major stumbling blocks to treatment in resource-constrained settings.

\section{What this study adds to the field}

This study addresses the two aspects of GDM that make it unique amongst the various forms of diabetes, i.e. the extremely tight glycemic control required and the short duration of hyperglycemia that renders conventional biomarkers such as glycated hemoglobin (HbA1c) unsuitable for long-term monitoring of glycemic control. By formulating a glycemic score that accurately predicts fetal outcomes, and yet allows for some latitude in the degree of hyperglycemia, this study represents an important step in making the management of GDM more user-friendly and practicable in the developing world.

Key words: fetal outcomes, gestational diabetes mellitus, glycemic control, maternal hypoglycemia, predictor, prognostic marker

From the Department of Medicine, Kasturba Hospital, Manipal, Karnataka, India

Received: Oct. 02, 2013; Accepted: Apr. 20, 2014

Correspondence to: Dr. Sowjanya Naha, Department of Medicine, Kasturba Hospital, Manipal, Karnataka, India. Madav Nagar, Manipal - 576 104, Karnataka, India. Tel.: 91-8202922812. Fax: 91-8202571927; E-mail: dasaris2001@yahoo.com

DOI: $10.4103 / 2319-4170.131399$ 
$\mathrm{G}_{\mathrm{c}}^{\mathrm{e}}$ estational diabetes mellitus (GDM) is a state of carbohydrate intolerance with onset or first recognition during pregnancy. ${ }^{[1]}$ It can complicate $7-14 \%$ of all pregnancies. ${ }^{[2]}$ In addition to adversely impacting immediate maternal and fetal outcomes in the form of maternal mortality and fetal death and malformation respectively, ${ }^{[2]}$ GDM has also been linked with long-term sequelae including increased risk of development of type 2 diabetes in the mother ${ }^{[2]}$ and greater prevalence of cardio-metabolic risk factors in the child. ${ }^{[3]}$ These associations gain importance in the developed world where tight gestational glycemic control is regularly achieved, with a concomitantly low incidence of acute maternal and fetal complications. In contrast, in resource-constrained settings, it is often impracticable to achieve tight glycemic control; intensive self-monitoring of blood glucose (SMBG), which has been proven to improve glycemic control in diabetes, ${ }^{[4]}$ is often not feasible, and attempts to aggressively lower blood glucose levels can result in recurrent hypoglycemic episodes for the mother. Consequently, efforts to lower blood glucose can paradoxically lead to non-compliance with intensive therapy, resulting in unacceptably high blood glucose levels and an increased incidence of adverse maternal and fetal outcomes. The situation is further compounded by the high prevalence of GDM in developing countries like India. ${ }^{[5]}$

This study was designed to determine whether a more lenient strategy might also provide satisfactory maternal and fetal outcomes. A glycemic score was also formulated for prognostication of fetal outcomes.

\section{METHODS}

\section{Study setting}

Kasturba Medical College, Manipal is a major tertiary care hospital in southwestern India, with a catchment area corresponding to the district of Udupi in which it is situated and the neighboring districts of Uttara Kannada and Dakshina Kannada. The combined population of this region is nearly 4.36 million individuals.

A prospective non-interventional study was conducted on consecutive patients admitted with GDM between May 2007 and August 2009, after obtaining clearance from the institutional ethics committee (IEC). GDM was diagnosed in all patients with the 100-g oral glucose tolerance test (OGTT). ${ }^{[2]}$ After an overnight fast of at least $8 \mathrm{~h}$ following 3 days of unrestricted diet, a fasting blood sample was drawn for blood glucose estimation. The patient then consumed $100 \mathrm{~g}$ of oral glucose. Thereafter, blood samples were sequentially drawn at 1,2 , and $3 \mathrm{~h}$ for estimation of blood glucose. The cutoff values for fasting and postprandial blood glucose values in this test were $95 \mathrm{mg} / \mathrm{dl}, 180 \mathrm{mg} / \mathrm{dl}, 155 \mathrm{mg} / \mathrm{dl}$, and $140 \mathrm{mg} / \mathrm{dl}$, re- spectively. A diagnosis of GDM was made when any two of these values were exceeded.

All included patients then underwent at least three glucose profiles, comprising fasting blood glucose and 2-h postprandial blood glucose following breakfast, lunch, and dinner, performed within a period of $24 \mathrm{~h}$. Glucose profiles were performed at least 1 month apart to allow for the impact of dietary and pharmacologic interventions. Patients with pre-gestational diabetes and those patients with GDM in whom less than three glucose profiles had been performed were excluded from the study. Patients lost for follow-up prior to delivery were also excluded from the study.

All patients were treated with diet control, insulin and/or metformin at the discretion of the treating consultants. All included patients were also screened for common causes of fetal malformation including alcohol abuse, consumption of teratogenic medication, and Toxoplasmosis, Others including syphilis and Human Immunodeficiency virus, Rubella, Cytomegalovirus and Herpes simplex virus-2 (TORCH) infections, and found to be negative.

Established risk factors for GDM were documented for all patients. This included a history of diabetes among first-degree relatives and an unfavorable obstetric history. Previous history of gestational diabetes, macrosomia, fetal malformation, or fetal loss was considered unfavorable. Maternal age at the time of conception was also noted. Body mass index (BMI) was calculated for all patients based on documented weight in the first trimester to minimize alteration by pregnancy.

Glycemic control was estimated by performing glucose profiles. The mean values of all glucose profiles for a given patient were noted. Fasting blood glucose below $95 \mathrm{mg} / \mathrm{dl}$ and postprandial blood glucose below $120 \mathrm{mg} / \mathrm{dl}$ were considered controlled. A glycemic score was calculated based on the number of mean blood glucose values (one fasting and three postprandial) that were controlled, ranging from 0 (no blood glucose values controlled) to 4 (all four blood glucose values controlled). Patients were followed up till the time of delivery, at which point fetal outcomes were noted.

Fetal macrosomia and congenital anomalies were considered as adverse fetal outcomes for the purpose of the study. Macrosomia was defined as a birth weight greater than $3500 \mathrm{~g}$ (corresponding to the $90^{\text {th }}$ percentile in the Indian population). ${ }^{[6]}$ The primary maternal outcome was survival.

\section{Statistical analysis}

All data were analyzed using SPSS Statistics version 17.0 (SPSS Inc., Chicago, IL, USA). Continuous variables were presented as mean \pm standard deviation (SD). Independent sample $t$-test and Kruskal-Wallis test were used to compare the means of variables between various patient 
subsets. Fisher's exact test was used to compare the frequencies of complications between the subsets. Sensitivity and specificity of the glycemic score for fetal outcomes were determined using receiver operating characteristic (ROC) curve. Binomial logistic regression was performed to determine independent association of the glycemic score with fetal outcomes. The $p$ values less than 0.05 were considered to indicate statistical significance.

\section{RESULTS}

Ninety-four patients with GDM fulfilling the inclusion criteria were included in the study.

Evaluation of the established risk factors for development of $\mathrm{GDM}^{[7]}$ revealed the following data.

Mean maternal age at conception was $29.62 \pm 4.46$ years. $83 \%$ of the patients were older than 25 years. Mean maternal BMI was $25.51 \pm 3.54 \mathrm{~kg} / \mathrm{m}^{2} .68 .1 \%$ of the patients had a BMI $>23 \mathrm{~kg} / \mathrm{m}^{2} .78 .4 \%$ of the patients had an unfavorable obstetric history, excluding primigravidae. $29.8 \%$ patients had a positive family history of diabetes in a first-degree relative. Overall, $77.66 \%$ of the patients displayed two or more risk factors for GDM.

The primary maternal outcome of survival was achieved in all cases. The primary fetal outcomes of macrosomia and congenital anomalies were noted in 11 and 6 cases, respectively. Congenital anomalies encountered included two cases of ventricular septal defect, combined atrial septal defect and patent ductus arteriosus, bilateral cleft lip, congenital diaphragmatic hernia, and small left colon. In addition, one infant had respiratory distress syndrome and another had hyperbilirubinemia. There were no cases of stillbirth, seizures, jaundice, or hypoglycemia.

While individual components of the glucose profile did not correlate significantly with these outcomes, the glycemic score was significantly associated with adverse fetal outcomes $(p<0.001)$. Analysis by ROC curve [Figure 1] showed good sensitivity and specificity for fetal macrosomia $(78.3 \%$ and $81.8 \%$, respectively) with a glycemic score of 2 or less [area under curve (AUC) 0.768 ; odds ratio (OR), 11.17; 95\% Confidence Interval (CI), 2.58-48.35; $p<0.001]$.

A similar but weaker association [Figure 2] was obtained for congenital anomalies (sensitivity $73.9 \%$, specificity $66.7 \%$ ) with a glycemic score of 2 or less (AUC 0.765 ; OR, 2.22; 95\% CI, 0.71-6.92; $p=0.055$ ).

Factors such as age and BMI did not correlate significantly with fetal outcomes [Table 1].

Binomial logistic regression confirmed the glycemic score to be predictive of fetal outcome independent of these risk factors [Table 2]. Adjusted OR values for macrosomia and congenital anomalies were 2.14 (95\% CI, 1.16-3.95) and 2.24 (95\% CI, 1.01-5.01), respectively.

\section{DISCUSSION}

While the adverse impact of diabetes on pregnancy in terms of maternal and fetal outcomes is well established, the available evidence pertains principally to pregnancy in patients with pre-existing diabetes, i.e. pre-gestational diabetes. ${ }^{[8]}$ Although data available on gestational diabetes is limited, the devastating effects of pre-gestational diabetes on pregnancy have resulted in the application of rigorous glycemic control to patients with GDM as well. ${ }^{[9]}$

Of the various complications resulting from hyperglycemia in pregnancy, macrosomia is the only outcome strongly associated with GDM. ${ }^{[10]}$ Nevertheless, even macrosomia has been shown to be associated with maternal BMI rather than GDM itself, ${ }^{[11]}$ although such an association was not seen in our study.

As a result of this uncertainty over the impact of GDM on pregnancy, the cost-effectiveness of mass screening and treatment of GDM has been questioned, especially in resource-constrained settings. ${ }^{[12]}$

Despite the absence of definite evidence supporting intensive glycemic control in patients with GDM, complete cessation of screening and treatment for this condition, as advocated by certain studies, ${ }^{[12]}$ would still appear extreme, especially when considering that hyperglycemia is easily reversible with appropriate therapy. Although not a primary objective of our study, careful appraisal for risk factors for GDM revealed at least one risk factor in nearly $95 \%$ of the patients and two risk factors in over three quarters. This finding would suggest that screening of patients with risk factors for GDM might be a reasonable alternative to mass screening, at least in resource-constrained settings.

Our study also revealed a high incidence of macrosomia and fetal malformation in patients with GDM. This result

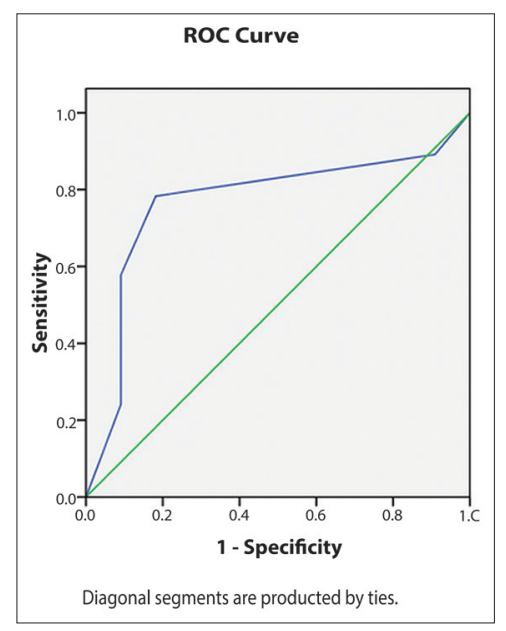

Figure 1: Receiver operating curve (ROC) demonstrating $78.3 \%$ sensitivity and $81.8 \%$ specificity for fetal macrosomia with a glycemic score of 2 or less [area under curve (AUC) 0.768 ; odds ratio (OR), 11.17; 95\% Confidence Interval (CI), 2.58-48.35; $p<0.001]$. 
was certainly confounded by the fact that our hospital is a tertiary referral center, and population-based studies are required for confirmation. Nevertheless, the finding is suggestive of the potential impact of GDM on pregnancy outcomes.

Current clinical practice guidelines recommend fasting blood glucose level below $95 \mathrm{mg} / \mathrm{dl}$ and postprandial blood glucose level below $120 \mathrm{mg} / \mathrm{dl}$ as the glycemic targets in women with GDM. ${ }^{[2]}$ Failure to achieve these targets with medical nutrition therapy (MNT) necessitates initiation of treatment with insulin in order to achieve similar targets. Inevitably, such intensive glycemic control involves frequent SMBG in order to forestall and prevent hypoglycemic episodes for the mother. SMBG is especially crucial in mothers experiencing emesis gravidarum, wherein unpredictable changes in caloric consumption require appropriate modification of insulin dosing. The psychological impact of such rigorous blood sampling can also be significant in patients who were otherwise healthy prior to conception. ${ }^{[10]}$

Needless to say, SMBG is often impracticable in daily clinical practice in developing countries due to a combination of financial constraints and ignorance of GDM in the population, resulting in patient non-compliance. Irregular monitoring of blood glucose can produce recurrent maternal hypoglycemic episodes, which can lead to further non-compliance to therapy and eventual patient dropout

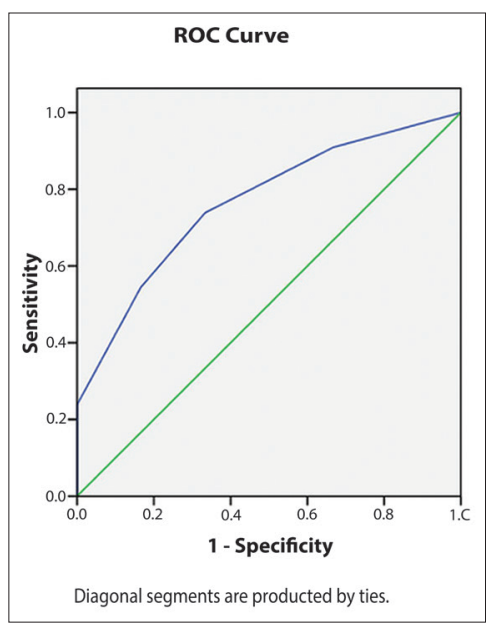

Figure 2: Receiver operating curve (ROC) demonstrating $73.9 \%$ sensitivity and $66.7 \%$ specificity for congenital anomalies with a glycemic score of 2 or less (AUC 0.765; OR, 2.22; 95\% CI, 0.71-6.92; $p=0.055)$. from follow-up. The absence of guidelines in such a situation presents the treating physician with an impossible choice - target intensive glycemic control and risk patient dropout, or maintain moderate glycemic control but risk fetal outcome. Indeed, even the term "moderate" here is nebulous, as there are no secondary targets available in the literature that can be aimed for when intensive glycemic control cannot be achieved.

Our study demonstrates that such a moderate approach may indeed be reasonable, wherein consistent achievement of three glycemic targets within the glucose profile appears sufficient to assure favorable fetal outcomes, while avoiding maternal hypoglycemia.

The formulation of the glycemic score which is easy to calculate and shows good sensitivity and specificity for fetal outcomes would also be beneficial in clinical practice, allowing for rapid prognostication, using readily available investigations. Utilization of an average of three glucose profiles provides a better picture of long-term glycemic control, correcting for acute fluctuations in glucose control that can alter a single glucose profile. In theory, this approach is similar to glycated hemoglobin (HbA1c) estimation, which has been shown in numerous trials to correlate with the complications of both type 1 and type 2 diabetes. Unfortunately, the relatively short duration of hyperglycemia in GDM precludes the usefulness of $\mathrm{HbA1c}$ as a marker of diabetic complications. Various studies have demonstrated the ineffectiveness of HbAlc as a diagnostic tool ${ }^{[13]}$ as well as a prognostic factor for GDM. Another biomarker, fructosamine, has also failed to show promise in patients with GDM. ${ }^{[14]}$ Conversely, the glycemic score used in this study correlated closely with fetal macrosomia and was also cost-effective as it did not require the performance of any additional biomarker assays.

In conclusion, the glycemic score is a simple, cost-effective, and effective prognostic marker of pregnancy outcomes in patients with GDM. Achievement of a target glycemic score of 3 assures favorable fetal outcomes while reducing the risk of maternal hypoglycemia, providing physicians with a secondary glycemic target for patients unwilling or unsuitable for intensive glycemic control and/or SMBG.

This study was limited by its sample size and a risk of bias due to the fact that it was hospital based in design. Larger population-based studies are required to further validate the glycemic score.

Table 1: Comparison of risk factors across patient subsets according to fetal outcome

\begin{tabular}{|c|c|c|c|c|}
\hline Risk factor & Healthy baby $(n=77)$ & Macrosomia $(n=11)$ & Congenital malformation $(n=6)$ & $p$ value \\
\hline Maternal age (years) & $29.72 \pm 4.6$ & $28.00 \pm 3.0$ & $31.67 \pm 4.6$ & 0.241 \\
\hline Maternal BMI $\left(\mathrm{kg} / \mathrm{m}^{2}\right)$ & $25.35 \pm 3.5$ & $26.99 \pm 4.1$ & $24.63 \pm 2.6$ & 0.464 \\
\hline Family history of diabetes $(\%)$ & $22(28.57 \%)$ & $4(57.14 \%)$ & $3(50.00 \%)$ & 0.417 \\
\hline Unfavorable obstetric history (\%) & $35(45.45 \%)$ & $3(42.86 \%)$ & $2(33.33 \%)$ & 0.556 \\
\hline Glycemic score $\leq 2(\%)$ & $32(41.56 \%)$ & $10(90.9 \%)$ & $5(83.3 \%)$ & 0.001 \\
\hline
\end{tabular}

Abbreviation: BMI: Body mass index 
Table 2: Binomial logistic regression with controlled variables and adjusted $p$ values

\begin{tabular}{llc}
\hline Fetal outcome & Variable & $\begin{array}{c}\text { Adjusted } \\
p \text { value }\end{array}$ \\
\hline Macrosomia & Maternal age $($ years $)$ & 0.175 \\
& Maternal BMI $\left(\mathrm{kg} / \mathrm{m}^{2}\right)$ & 0.341 \\
& Family history of diabetes & 0.952 \\
& Unfavorable obstetric history & 0.824 \\
& Glycemic score $\leq 2$ & 0.015 \\
Congenital malformation & Maternal age $($ years) & 0.164 \\
& Maternal BMI $\left(\mathrm{kg} / \mathrm{m}^{2}\right)$ & 0.255 \\
& Family history of diabetes & 0.169 \\
& Unfavorable obstetric history & 0.621 \\
& Glycemic score $\leq 2$ & 0.049
\end{tabular}

Abbreviation: BMI: Body mass index

\section{REFERENCES}

1. Metzger BE, Coustan DR. Summary and recommendations of the fourth international workshop-conference on gestational diabetes mellitus. Diabetes Care 1998;21 Suppl 2:B161-7.

2. American Diabetes Association. Gestational diabetes mellitus. Diabetes Care 2003;26 Suppl 1:S103-5.

3. Patel S, Fraser A, Davey Smith G, Lindsay RS, Sattar N, Nelson SM, et al. Associations of gestational diabetes, existing diabetes, and glycosuria with offspring obesity and cardiometabolic outcomes. Diabetes Care 2012;35:63-71.

4. Huang IC, Wang PW, Liu RT, Tung SC, Chen JF, Kuo MC, et al. The influence of self-monitoring blood glucose frequency on the oscillation of hemoglobin A1c and chronic complications. Chang Gung Med J 2012;35:46-53.
5. Ramachandran A, Snehalatha C, Shyamala P, Vijay V, Viswanathan M. Prevalence of diabetes in pregnant women - a study from southern India. Diabetes Res Clin Pract 1994;25:71-4.

6. Balaji V, Balaji M, Anjalakshi C, Cynthia A, Arthi T, Seshiah V. Diagnosis of gestational diabetes mellitus in Asian-Indian women. Indian J Endocrinol Metab 2011;15:187-90.

7. Cheung NW, Wasmer G, Al-Ali J. Risk factors for gestational diabetes among Asian women. Diabetes Care 2001;24:955-6.

8. Enkin M, Keirse MJ, Neilson J, Crowther C, Duley L, Hodnett E, editors. A guide to effective care in pregnancy and childbirth. $3^{\text {rd }} \mathrm{ed}$. Oxford: Oxford University Press; 2000.

9. Tuffnell DJ, West J, Walkinshaw SA. Treatments for gestational diabetes and impaired glucose tolerance in pregnancy. Cochrane Database Syst Rev 2003;3:CD003395.

10. Jarrett RJ, Castro-Soares J, Dornhorst A, Beard RW, Castro-Soares J. The concept of gestational diabetes was popularised before considerations of evidence based medicine came on the scene. BMJ 1997;315:736-9.

11. Green JR, Schumacher LB, Pawson IG, Partridge JC, Kretchmer N. Influence of maternal body habitus and glucose tolerance on birth weight. Obstet Gynecol 1991;78:235-40.

12. Ramtoola S, Home P, Damry H, Husnoo A, Ah-Kion S. Gestational impaired glucose tolerance does not increase perinatal mortality in a developing country: A cohort study. BMJ 2001;322:1025-6.

13. Agarwal MM, Dhatt GS, Punnose J, Koster G. Gestational diabetes: A reappraisal of HbA1c as a screening test. Acta Obstet Gynecol Scand 2005;84:1159-63.

14. Shefali AK, Kavitha M, Deepa R, Mohan V. Pregnancy outcomes in pre-gestational and gestational diabetic women in comparison to non-diabetic women-A prospective study in Asian Indian mothers (CURES-35). J Assoc Physicians India 2006;54:613-8. 